\title{
Avaliação da gamificação na área educacional: um mapeamento sistemático
}

\author{
Aline Nunes $\operatorname{Ogawa}^{1}$, Ana Carolina Tomé Klock ${ }^{1,2}$, Isabela Gasparini ${ }^{1,2,3}$ \\ ${ }^{1}$ Departamento de Ciência da Computação, ${ }^{2}$ PPGCA, ${ }^{3}$ PPGECMT \\ ${ }^{1,2,3}$ Universidade do Estado de Santa Catarina (UDESC) - Joinville, SC - Brasil \\ alinenogawa@hotmail.com, actklockgmail.com, isabela.gasparini@udesc.br
}

\begin{abstract}
Gamification is a technique that extracts elements from games and reuses them in other contexts, being applied in educational area to motivate and engage students. The aim of this paper is to find if there are any works in the literature that make the comparison and the assessment of educational environments with and without gamification. Thus, a systematic mapping was conducted and, from the 832 items returned, 24 were analyzed after they meet the selection criteria. As a result, it was observed that most of the work compares and evaluates the environments through the performance, satisfaction and interaction of students. These works analyzed different students' activities, such as performing tests, exercises and satisfaction surveys.
\end{abstract}

Resumo. Gamificação é uma técnica que extrai elementos de jogos e os reutiliza em outros contextos, sendo aplicada na área educacional para motivar e engajar os estudantes. O objetivo deste artigo é verificar se existem trabalhos na literatura que fazem a comparação e avaliação de ambientes educacionais com e sem gamificação. Desta forma, foi realizado um mapeamento sistemático e, dos 832 artigos retornados, 24 foram analisados após atenderem aos critérios de seleção. Como resultado, foi possível observar que a maioria dos trabalhos compara e avalia os ambientes por meio do desempenho, da satisfação e da interação dos estudantes. Os trabalhos analisaram as diferentes atividades executadas pelos estudantes, como a realização de testes, exercícios e pesquisas de satisfação.

\section{Introdução}

A educação necessita de novas estratégias para atrair interesse dos estudantes que se mostram desinteressados pelos métodos passivos de ensino e aprendizagem utilizados na maioria das escolas (Fardo, 2013). Atualmente, muito tem se investido na área de informática na educação para encontrar tecnologias que possam ser aplicadas no processo de ensino e aprendizagem com o intuito de motivar e engajar os estudantes.

Uma tecnologia amplamente estudada e aplicada no processo de aprendizagem é a gamificação. A gamificação é o termo utilizado para definir a utilização de elementos e design de jogos em contextos não lúdicos a fim de estimular e motivar seus usuários a alcançarem algum objetivo específico, a mudarem seu comportamento ou a desenvolverem uma nova habilidade (Werbach; Hunter, 2012; Deterding et al., 2011).

$\mathrm{O}$ uso de gamificação pode ter um grande potencial na educação tradicional (em sala de aula), onde existem estudantes desmotivados e professores que não conseguem envolvê-los nas atividades de aprendizagem (De-Marcos et al., 2014). A gamificação também pode ser utilizada em ambientes educacionais on-line, mas em muitos casos a falta de contato direto com um professor pode prejudicar a interação do estudante. A 
V Congresso Brasileiro de Informática na Educação (CBIE 2016)

Anais do XXVII Simpósio Brasileiro de Informática na Educação (SBIE 2016)

gamificação possui um amplo conjunto de abordagens de ensino e aprendizagem e, quando aplicada de forma eficaz, pode ajudar o estudante na aquisição de uma nova habilidade ao mesmo tempo em que aumenta sua motivação para aprender (Johnson et al., 2013). Dentre os elementos de jogos comumente utilizados em ambientes educacionais, podem ser citados: feedback constante, desafios, competição, conquistas, recompensas, pontos, medalhas, missões, personalização, regras, narrativa, níveis e rankings (Bissolotti; Nogueira; Pereira, 2014; Klock et al., 2014).

Desta forma, o objetivo desse artigo é identificar como é realizada a avaliação da gamificação no contexto educacional. Este trabalho realiza um mapeamento sistemático para identificar como é realizado o processo de avaliação dos ambientes educacionais e quais os pontos positivos e negativos da utilização da gamificação. Para isso, este trabalho está estruturado como segue: a seção 2 apresenta o processo do mapeamento, onde são definidas as questões de pesquisa, o argumento e os mecanismos de busca utilizados, além da delimitação dos critérios de inclusão e exclusão. A seção 3 realiza as análises, respondendo cada questão de pesquisa. Por fim, na seção 4 são apresentadas as conclusões do mapeamento realizado.

\section{Processo do Mapeamento}

Petersen, Vakkalanka e Kuzniarz (2015) definem mapeamento sistemático como um processo que classifica e contabiliza as contribuições existentes em uma determinada área que possam dar uma visão geral da área de pesquisa. Para a elaboração de um mapeamento sistemático, é necessário definir as questões de pesquisa, o processo de busca (que envolve a definição do argumento de busca a partir das questões de pesquisa e a seleção dos mecanismos de busca a serem utilizados) e o processo de seleção (onde são definidos os critérios de inclusão e exclusão) dos artigos encontrados. Os artigos que passarem pela etapa de seleção têm seus dados extraídos para analisar e, posteriormente, responder as questões de pesquisa definidas (Petersen, Vakkalanka e Kuzniarz, 2015; Klock et al., 2015).

\subsection{Questões de Pesquisa}

Para identificar como ocorre a avaliação da gamificação no contexto educacional, formam definidas as seguintes Questões de Pesquisa (QP):

- $\mathbf{Q P}_{1}$ : Como é realizada a comparação e a avaliação dos ambientes educacionais com e sem gamificação?

- $\mathbf{Q P}_{2}$ : Quais atividades fazem parte do processo de avaliação dos ambientes educacionais?

- $\mathbf{Q P}_{3}$ : Quais são os pontos positivos e negativos da utilização de ambientes educacionais com gamificação?

\subsection{Processo de Busca}

Com as questões de pesquisa, foi definido o argumento de busca a ser utilizado. Para isso, as questões de pesquisa foram detalhadas em três grandes categorias de palavraschaves: relacionadas à gamificação (e.g., gamification, gamified, gamifying), relacionadas à educação (e.g., education, learning, educational) e relacionadas ao processo de avaliação (e.g., improving, evaluate, case study, test, validation, assessment). Assim, foram buscados artigos que atendessem o argumento de busca: "(gamification OR gamified OR gamif*) AND (education* OR learning) AND (improving OR evaluat* OR "case study" OR assessment OR validation OR 
V Congresso Brasileiro de Informática na Educação (CBIE 2016)

Anais do XXVII Simpósio Brasileiro de Informática na Educação (SBIE 2016)

experiment* OR test* OR "empirical study")", no título, resumo ou palavras-chave, sendo * equivalente ao caractere coringa.

Os mecanismos de busca acadêmicos utilizados foram: ACM Digital Library, Science Direct (SDirect), IEEE Xplore, Scopus, SpringerLink e Web of Science (WoS). A escolha desses mecanismos foi feita a partir da observação de outros mapeamentos sistemáticos referentes à gamificação, como o de Sousa Borges et al. (2014) e Klock et al. (2015). A busca foi realizada em novembro de 2015, filtrando apenas artigos publicados a partir de 2010 (ano em que a gamificação ganhou destaque (Burke, 2014)) e no idioma em inglês (idioma predominante nos mecanismos). Ainda, como não é possível utilizar o caractere coringa na ACM Digital Library, foi necessário adaptar o argumento de busca. A Tabela 1 apresenta a quantidade de artigos retornados em cada mecanismo de busca acadêmico.

Tabela 1. Quantidade de artigos retornados pelos Mecanismos

\begin{tabular}{ll} 
Mecanismo de Busca & Quantidade \\
\hline ACM Digital Library & 97 \\
Science Direct & 33 \\
IEEE Xplore & 212 \\
Scopus & 264 \\
SpringerLink & 193 \\
Web of Science & 33 \\
\hline Total & $\mathbf{8 3 2}$
\end{tabular}

Dessa forma, foi possível obter 832 artigos que apresentam a avaliação da gamificação no contexto educacional durante o processo de busca. Os artigos identificados são analisados pelo processo de seleção para avaliar sua pertinência em relação ao objetivo do trabalho.

\subsection{Processo de Seleção}

Os critérios de inclusão e exclusão foram definidos com base nas questões de pesquisa para tornar o resultado do mapeamento sistemático mais assertivo. Além da definição do período de publicação e do idioma, foram definidos os seguintes critérios de inclusão:

- Artigos disponíveis para download;

- Artigos completos (foram considerados artigos com mais de 4 páginas); Quanto aos critérios de exclusão, tem-se:

- Artigos duplicados;

- Artigos não relacionados com a gamificação;

- Artigos não relacionados com a área educacional;

- Artigos que não descrevem o processo de avaliação do ambiente educacional com gamificação por meio da comparação com ambientes educacionais sem gamificação.

Assim, os artigos que não atenderam qualquer um dos critérios foram removidos. 
V Congresso Brasileiro de Informática na Educação (CBIE 2016)

Anais do XXVII Simpósio Brasileiro de Informática na Educação (SBIE 2016)

Tabela 2. Artigos retornados a cada critério durante o processo de seleção

\begin{tabular}{l|cccccc|c}
\hline & ACM DL & SDirect & IEEE & Scopus & Springer & WoS & Total \\
\hline Artigos & 97 & 33 & 212 & 264 & 193 & 33 & 832 \\
retornados & 96 & 33 & 208 & 101 & 191 & 20 & 649 \\
Disponíveis & 89 & 33 & 178 & 97 & 170 & 20 & 587 \\
Completos & & & & & 504 \\
\hline Remoção dos artigos duplicados & & & 355 \\
Artigos relacionados com gamificação & \\
Artigos relacionados com a área educacional \\
Artigos que fazem comparação de ambientes com e sem gamificação & 183 \\
\hline
\end{tabular}

Conforme a Tabela 2, dos 832 artigos retornados, apenas 587 artigos possuíam os critérios de inclusão (i.e., artigos disponíveis para download e completos) e, após a remoção dos artigos duplicados, restaram 504. Desses, foram identificados 355 contemplavam a gamificação, sendo que 183 estavam dentro do contexto educacional, mas apenas 24 descreviam o processo avaliativo por meio de comparação entre os ambientes. A lista com os 24 artigos está disponível em https://goo.gl/udGfMw.

\section{Análise e Discussão}

Dos 24 artigos resultantes, foram extraídos dados para responder as questões de pesquisa e identificar o processo de avaliação dos ambientes educacionais com gamificação e quais seus pontos positivos e negativos, tais como: ano de publicação, mecanismo de busca, veículo de publicação (e.g., periódico, evento), assuntos ou disciplinas abordados (e.g., computação, matemática, ciências, saúde), existência de questão de pesquisa ou hipótese, as comparações realizadas nos trabalhos, elementos de jogos, abordagem (e.g., between-subject, within-subject), período de realização do experimento (e.g., mesmo período, períodos distintos), critério de divisão dos grupos, quantidade de participantes, duração do experimento, atividades (e.g., exercícios, tarefas, questionário de satisfação, teste, fórum de discussão, projeto e download de materiais), métricas e resultados.

Todos os trabalhos encontrados foram publicados entre 2013 e 2016, sendo 9 em 2013, 5 em 2014, 8 em 2015 e 2 já disponíveis de 2016. Em relação ao mecanismo de busca de origem, 7 dos artigos foram disponibilizados pelo mecanismo IEEE Xplore, 6 pelo ACM Digital Library, 10 pelo Scopus e 2 pelo Science Direct. Desses, 15 foram publicados em eventos e 9 publicados em periódicos, conforme ilustra o Gráfico 1.

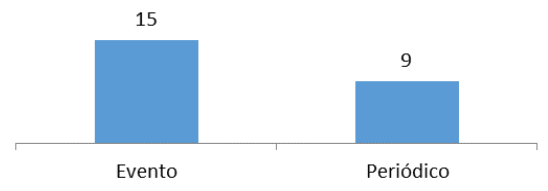

\section{Gráfico 1. Artigos por veículo de publicação}

Em relação aos assuntos ou disciplinas abordados pelos ambientes educacionais, foi verificado que a grande maioria aplica gamificação em disciplinas relacionadas com Computação (e.g., algoritmos, estrutura de dados, computação gráfica, conceitos básicos, desenvolvimento de jogos e de software). Porém, alguns se relacionam com a Matemática (e.g., fórmulas e problemas matemáticos), as Ciências (e.g., conceitos de ciências ecológicas), a Saúde (e.g., doenças), a Comunicação e Letras (e.g., vocabulário), conforme o Gráfico 2. 


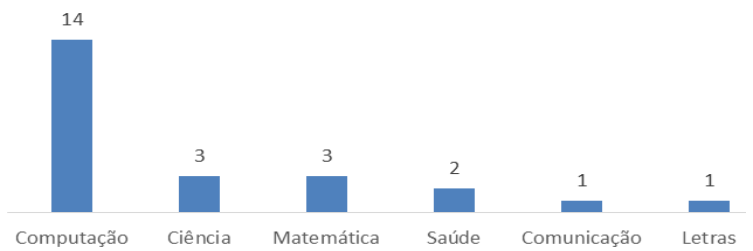

Gráfico 2. Artigos por assuntos abordados pelo ambiente educacional

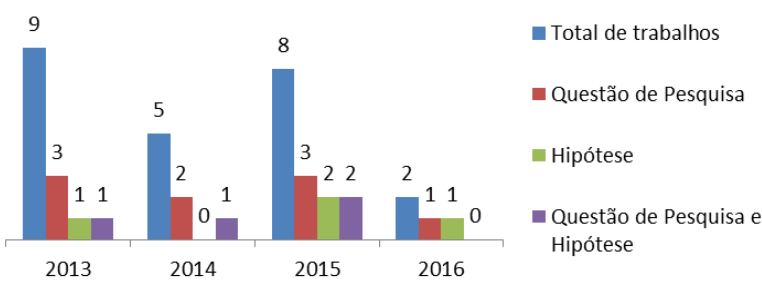

Gráfico 3. Artigos que possuem questão de pesquisa e hipótese

Quanto a existência de questão de pesquisa ou hipótese nos trabalhos analisados, percebe-se que existem trabalhos que não apresentam nem um ou outro, conforme o Gráfico 3. Porém, é perceptível que, com os passar dos anos, os autores têm se preocupado mais com a descrição dos mesmos. Em 2013, apenas 55,6\% dos trabalhos possuíam questão de pesquisa ou hipótese, 60\% dos trabalhos publicados em 2014, $87,5 \%$ dos trabalhos de 2015, por fim, todos os trabalhos já publicados em 2016 possuíam questão de pesquisa ou hipótese.

Os 24 artigos realizaram um experimento utilizando gamificação em pelo menos um dos seus trabalhos. Observando o Gráfico 4, é possível verificar que a maioria (22 dos trabalhos) realiza a comparação de ambientes com e sem gamificação. Destacam-se também os trabalhos que, além de fazer com e sem gamificação, realizam outras comparações. Por exemplo, o trabalho de Su e Cheng (2015) compara com gamificação (utilizando um aplicativo mobile) e sem gamificação (com e sem aplicativo), enquanto Krause et al. (2015) comparam gamificação (com e sem elementos sociais) e sem gamificação. Porém, também há comparações da gamificação com outros métodos (redes sociais e heatmap), respondendo assim a $\mathbf{Q} \mathbf{P}_{\mathbf{1}}$.

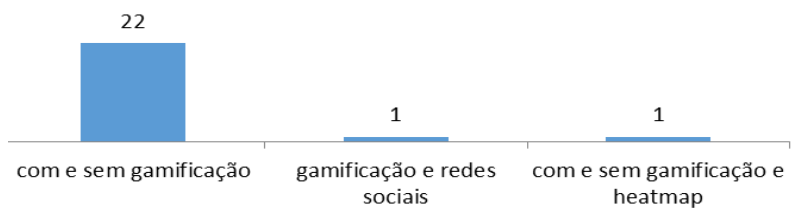

\section{Gráfico 4. Comparações realizadas pelos trabalhos}

Entre os ambientes educacionais utilizados, a maioria (15 dos trabalhos) aplica a gamificação em ambientes e-learning, mas há também páginas web, softwares, aplicativos e jogos. Desses ambientes e-learning, o Moodle foi utilizado por 4 trabalhos e o Blackboard por 2, enquanto os demais ambientes foram utilizados apenas uma vez. Ainda, 3 trabalhos não citam o ambiente avaliado.

Em relação aos elementos de jogos, a quantidade utilizada nos trabalhos é 1 elemento em 3 trabalhos, 2 em 6 trabalhos, 3 em 6 trabalhos, 4 em 6 trabalhos e 5 elementos em 2 trabalhos. Ainda há 1 trabalho que não informou. Os elementos mais utilizados pela gamificação são: medalhas (presente em 20 artigos), ranking (presente em 16 artigos), pontos (presente em 13 artigos) e níveis (presente em 7 artigos), conforme detalhado no Gráfico 5.

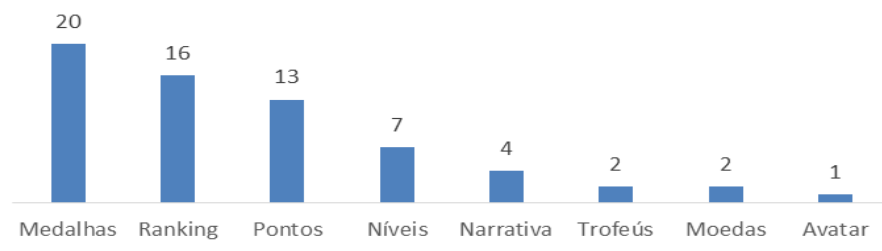

Gráfico 5. Elementos de jogos aplicados nos ambientes 
Quanto a aplicação do experimento, apenas o trabalho de Amriani et al. (2013) utiliza a abordagem within-subject para comparar os ambiente com e sem gamificação, ou seja, todos os participantes utilizam os dois ambientes. Os outros 23 trabalhos utilizam a abordagem between-subject, ou seja, grupos com quantidade similares de participantes são testados em diferentes condições ou ambientes. Desses trabalhos, 17 deles ocorreram no mesmo período e 7 em períodos distintos (i.e., dias, semestres ou anos diferentes). Em relação aos critérios de divisão dos grupos, a maioria é definida de modo aleatório (aplicado em 9 trabalhos), seguida pela separação por turmas (turmas distintas da mesma instituição - aplicado em 6 trabalhos) e de forma voluntária (o estudante pode optar por qual grupo participar - aplicado em 5 trabalhos). Além desses, 4 trabalhos não citam qual o critério utilizado.

Há uma grande variação da quantidade de estudantes nos experimentos realizados. A maioria (16 trabalhos) envolve entre 16 e 107 participantes, conforme mostra o Gráfico 6. Desse, Três trabalhos têm entre 16 e 30 participantes, três trabalhos entre 31 e 60 participantes, cinco trabalhos entre 61 e 90 participantes e sete entre 91 e 107 participantes. Há 4 trabalhos entre 196 e 371 participantes e 3 trabalhos com mais de 500 participantes. O trabalho de Atalli e Arieli-Atalli (2015) o maior deles, realizando dois experimentos: o primeiro com 1218 participantes e o segundo com 693, totalizando 1911 participantes, e o de Pedro et al. (2015) o que possui a menor quantidade de participantes, apenas 16 participantes. O trabalho de O'Donavan, Gain e Marais (2013) foi o único que informou apenas a quantidade de participantes do grupo com gamificação (90 participantes).

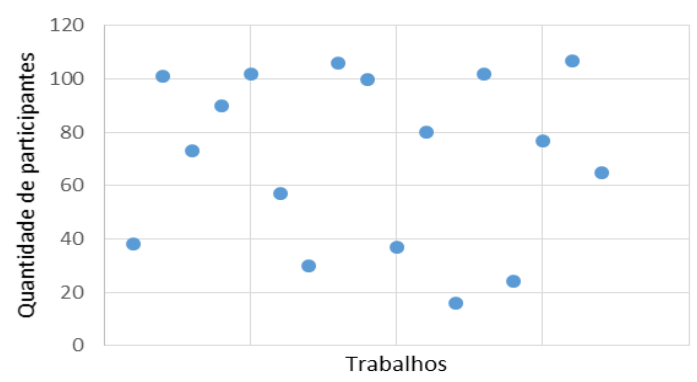

\section{Gráfico 6. Quantidade de participantes por artigo na faixa com mais trabalhos}

Em relação à duração dos experimentos, 6 trabalhos tiveram duração de até um mês, 4 trabalhos entre um mês e meio e dois meses, 4 trabalhos de um semestre e 4 trabalhos de um ano. Apenas o trabalho de Hew et al. (2016) teve duração distinta em seus experimentos, ocorrido em períodos distintos. $\mathrm{O}$ primeiro experimento durou três dias, enquanto o segundo, dezoito dias. O Gráfico 7 apresenta a distribuição do período de duração dos experimentos em meses por artigo. Além disso, 5 trabalhos não citam o período de aplicação do experimento.

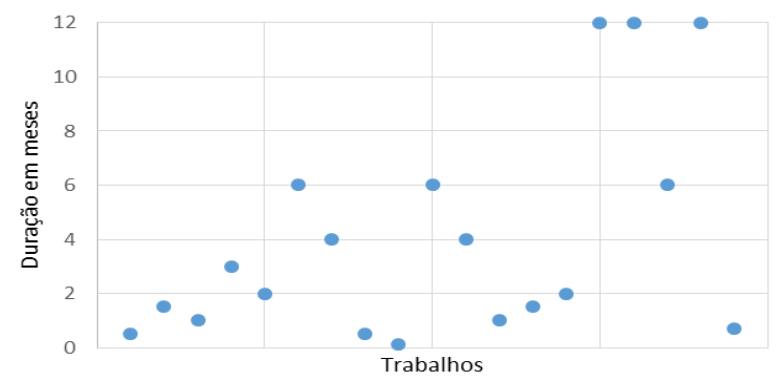

Gráfico 7. Duração dos experimentos em meses 
V Congresso Brasileiro de Informática na Educação (CBIE 2016)

Anais do XXVII Simpósio Brasileiro de Informática na Educação (SBIE 2016)

O processo de avaliação dos trabalhos é composto, geralmente, por 2 ou 3 atividades analisadas, como pode ser observado na Tabela 3. A maioria dos experimentos incluíram as atividades de exercícios ou tarefas, pesquisas de satisfação e teste para avaliar a gamificação, o que responde a $\mathbf{Q} \mathbf{P}_{\mathbf{2}}$.

Para complementar a avaliação da gamificação, 15 trabalhos obtiveram dados qualitativos. Desses, 11 trabalhos utilizam Escala de Likert (onde 10 utilizaram a escala de cinco pontos e 1 utilizou de cinco e sete pontos em questões distintas), 1 trabalho utilizou questões abertas e 3 trabalhos citam a avaliação qualitativa, mas não explicam como o processo foi realizado.

Tabela 3. Atividades utilizadas no processo avaliativo dos ambientes

\begin{tabular}{|c|c|c|c|c|c|c|c|c|}
\hline Autores & $\begin{array}{l}\text { Exercícios } \\
\text { ou Tarefas }\end{array}$ & $\begin{array}{l}\text { Questionário } \\
\text { de Satisfação }\end{array}$ & Teste & $\begin{array}{l}\text { Teste } \\
\text { final }\end{array}$ & $\begin{array}{l}\text { Fórum de } \\
\text { discussão }\end{array}$ & $\begin{array}{c}\text { Download } \\
\text { de materiais }\end{array}$ & Projeto & \\
\hline Amriani et al. (2013) & $\mathrm{x}$ & & $\mathrm{x}$ & & $\mathrm{x}$ & & & 3 \\
\hline Barata et al. (2013a) & & $\mathrm{x}$ & & $\mathrm{x}$ & $\mathrm{x}$ & $\mathrm{x}$ & & 4 \\
\hline Barata et al. (2013b) & & $\mathrm{x}$ & & $\mathrm{x}$ & $\mathrm{x}$ & $\mathrm{x}$ & & 4 \\
\hline Browne, Anand (2013) & $\mathrm{x}$ & $\mathrm{x}$ & & & & & & 2 \\
\hline Denny (2013) & $\mathrm{x}$ & $\mathrm{x}$ & & & & & & 2 \\
\hline Dominguez et al. (2013) & $\mathrm{x}$ & $\mathrm{x}$ & $\mathrm{x}$ & $\mathrm{x}$ & $\mathrm{x}$ & & & 5 \\
\hline Hakulinen, Auvinen, Korhonen (2013) & $\mathrm{x}$ & & & $\mathrm{x}$ & & & $\mathrm{x}$ & 3 \\
\hline O'Donovan, Gain, Marais (2013) & $\mathrm{x}$ & $\mathrm{x}$ & $\mathrm{x}$ & $\mathrm{x}$ & $\mathrm{x}$ & & & 5 \\
\hline Su, Cheng (2013) & $\mathrm{x}$ & $\mathrm{x}$ & $\mathrm{x}$ & & & & & 3 \\
\hline Buisman, Eekelen (2014) & $\mathrm{x}$ & & & & & & & 1 \\
\hline de-Marcos et al. (2014) & $\mathrm{x}$ & $\mathrm{x}$ & $\mathrm{x}$ & $\mathrm{x}$ & $\mathrm{x}$ & & & 5 \\
\hline Faghihi et al. (2014) & $\mathrm{x}$ & & $\mathrm{x}$ & & & & & 2 \\
\hline Filsecker, Hickey (2014) & $\mathrm{x}$ & & $\mathrm{x}$ & & & & & 2 \\
\hline Vaibhav, Gupta (2014) & $\mathrm{x}$ & $\mathrm{x}$ & $\mathrm{x}$ & $\mathrm{x}$ & & & & 4 \\
\hline Anderson, Nash, McCauley (2015) & $\mathrm{x}$ & $\mathrm{x}$ & & & & & & 2 \\
\hline Atalli, Arieli-Atalli (2015) & $\mathrm{x}$ & $\mathrm{x}$ & $\mathrm{x}$ & & & & & 3 \\
\hline Auvinen, Hakulinen, Malmi (2015) & $\mathrm{x}$ & $\mathrm{x}$ & & & & & & 2 \\
\hline Hanus, Fox (2015) & & $\mathrm{x}$ & $\mathrm{x}$ & $\mathrm{x}$ & & & & 3 \\
\hline Krause et al. (2015) & $\mathrm{x}$ & & $\mathrm{x}$ & $\mathrm{x}$ & & & & 3 \\
\hline Laskowski (2015) & $\mathrm{x}$ & & $\mathrm{x}$ & & & & $\mathrm{x}$ & 3 \\
\hline Pedro et al. (2015) & $\mathrm{x}$ & $\mathrm{x}$ & & & & & & 2 \\
\hline Su, Cheng (2015) & $\mathrm{x}$ & $\mathrm{x}$ & $\mathrm{x}$ & & & & & 3 \\
\hline Gonzalez et al. (2016) & $\mathrm{x}$ & & $\mathrm{x}$ & & & & & 2 \\
\hline \multirow[t]{2}{*}{ Hew et al. (2016) } & $\mathrm{x}$ & $\mathrm{x}$ & $\mathrm{x}$ & & $\mathrm{x}$ & $\mathrm{x}$ & & 5 \\
\hline & 21 & 16 & 15 & 9 & 7 & 3 & 2 & \\
\hline
\end{tabular}

As métricas utilizadas para a avaliação estão relacionadas às atividades descritas na Tabela 3 e às características pessoais dos estudantes. Sobre as atividades, a maioria das métricas está relacionada às notas/pontuações em exercícios, tarefas e testes (19 trabalhos), conclusão dos exercícios ou das tarefas (10 trabalhos) e acertos nos exercícios ou tarefas ( 7 trabalhos), conforme detalhado no Gráfico 8.

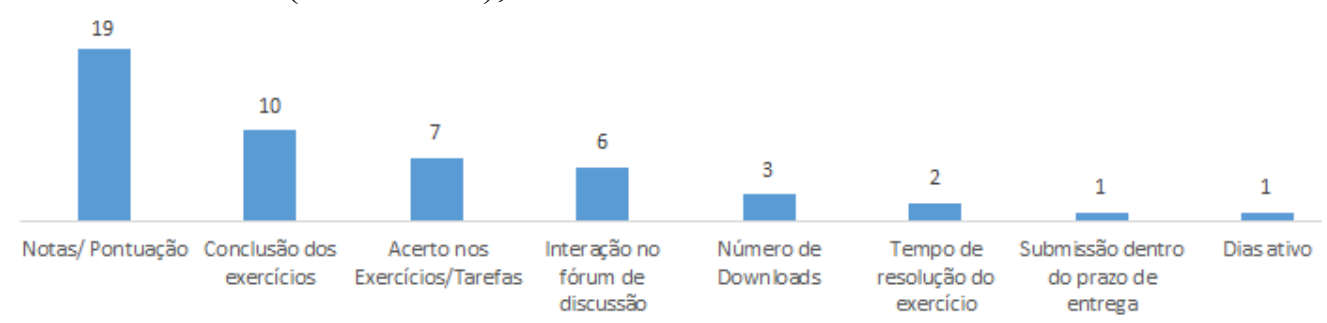

Gráfico 8. Métricas referentes ao processo avaliativo

Sobre as características pessoais dos estudantes, as métricas utilizadas são motivação (11 trabalhos), grau de satisfação (7 trabalhos), engajamento (6 trabalhos) e assiduidade (5 trabalhos), conforme ilustra o Gráfico 9. 
V Congresso Brasileiro de Informática na Educação (CBIE 2016)

Anais do XXVII Simpósio Brasileiro de Informática na Educação (SBIE 2016)

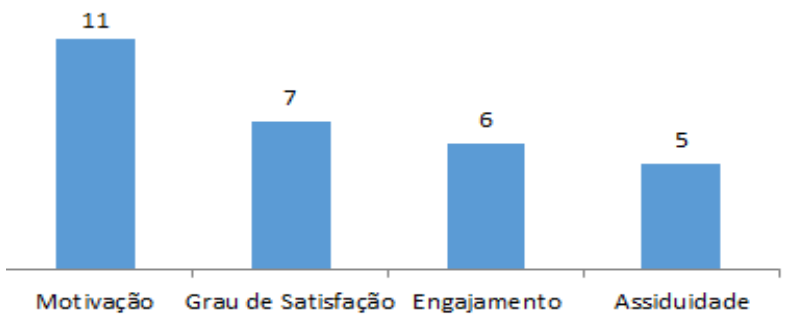

Gráfico 9. Métricas referentes a características pessoais

Quanto aos resultados obtidos, os trabalhos que compararam o ambiente com e sem gamificação, o trabalho de Amriani et al. (2013) mostrou o impacto na participação com a adição e remoção da gamificação. Na primeira semana, o grupo A utilizou o ambiente com gamificação e o grupo B sem gamificação. Na semana seguinte, houve a remoção da gamificação no grupo $\mathrm{A}$ e a adição de gamificação no grupo $\mathrm{B}$. Essa mudança resultou em um aumento não significativo da participação do grupo $\mathrm{B}$, porém reduziu significativamente a participação do grupo A.

A Tabela 4 apresenta de uma forma visual os resultados obtivos por cada trabalho durante a comparação entre os ambientes com e sem gamificação, informando se o resultado em alguns aspectos melhorou $(+)$, piorou $(-)$ ou se foi similar $(=)$ com o uso da gamificação, sendo que os campos destacados (mais escuros) apresentam diferenças estatísticamente significativas. Os aspectos que tiveram melhores resultados com a utilização da gamificação foram: motivação (7 trabalhos), e acerto nos exercícios ou tarefas, assiduidade, satisfação e engajamento (4 trabalhos cada). Nota/pontuação foi os aspecto com mais resultados positivos (12 trabalhos) e negativos ( 3 trabalhos).

Tabela 4. Resultado da comparação de ambientes com e sem gamificação

\begin{tabular}{|c|c|c|c|c|c|c|c|c|c|c|c|c|c|c|c|c|c|c|c|c|c|}
\hline 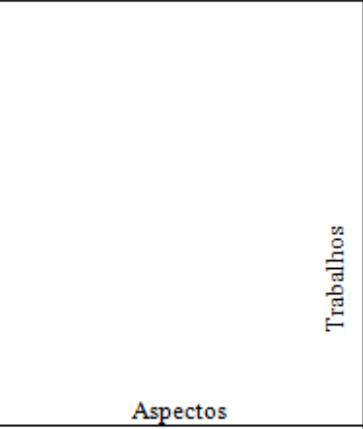 & 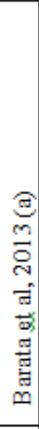 & 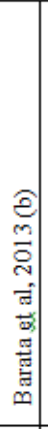 & 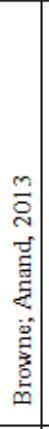 & 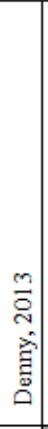 & 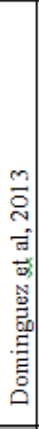 & 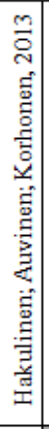 & 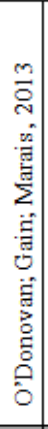 & 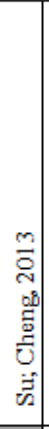 & 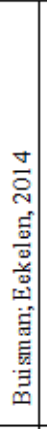 & 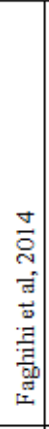 & 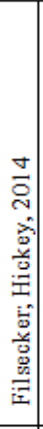 & 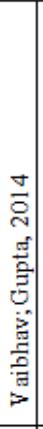 & 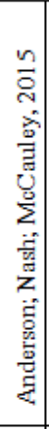 & 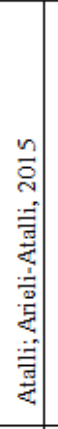 & 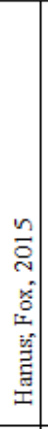 & 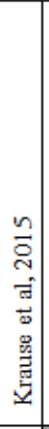 & 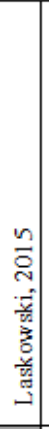 & 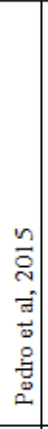 & 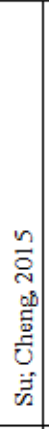 & 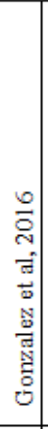 & 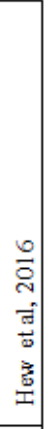 \\
\hline Nota/Pontuação & $=$ & $=$ & & & + & + & + & + & + & + & + & + & & & - & + & - & $=$ & & + & + \\
\hline Motivação & + & + & & & - & & + & & + & & $=$ & & & & - & & & & + & + & + \\
\hline Engajamento & + & + & & & - & & + & & + & & $=$ & & & & - & & & & & & \\
\hline Acerto nos exercícios/tarefas & & & & + & & + & & & & & & & & - & & + & & $=$ & & & \\
\hline Satisfação & + & & + & & & & & + & & & & & & & - & & & & + & & \\
\hline Assiduidade & + & + & & & & & & + & & & & & & & & & + & & & & \\
\hline Conclusão dos exercícios/tarefas & & & & + & - & + & & & & & & & + & & & & & & & & \\
\hline Participação no fónum & + & + & & & - & & & & & & & & & & & & & & & & + \\
\hline Download de materiais & + & + & & & & & & & & & & & & & & & & & & & + \\
\hline Teste final & & & & & - & & & & & & & + & & & - & + & & & & & \\
\hline Tempo de resposta & & & & & & & & & & & & & & + & & & & & & & \\
\hline Dias ativos & & & & + & & & & & & & & & & & & & & & & & \\
\hline Prazo de entrega & & & & & & + & & & & & & & & & & & & & & & \\
\hline
\end{tabular}

O trabalho de Su e Cheng (2015) compara o ambiente com gamificação (com aplicativo mobile) e sem gamificação (com e sem aplicativo). O experimento obteve em maior interação e melhor resultado utilizando o aplicativo com gamificação. Krause et al. (2015) comparou ambientes com e sem gamificação. A condição com gamificação foi analisada com e sem condição social, ou seja, o estudante poderia escolher o seu adversário ou o ambiente o escolheria de maneira aleatória. Como resultado, os autores 
V Congresso Brasileiro de Informática na Educação (CBIE 2016)

Anais do XXVII Simpósio Brasileiro de Informática na Educação (SBIE 2016)

observaram um período de retenção do conteúdo significativamente maior nos grupos com gamificação, sendo melhor com a condição social. $O$ grupo de gamificação com condição social possui precisão significativamente maior nas respostas, e os dois grupos de gamificação não possuem diferenças significativas entre si. Por fim, as condições com gamificação obtiveram médias maiores do que a condição sem gamificação no teste final, apesar de não ser significativa.

O trabalho de De-Marcos et al. (2014) compara a rede social com ambientes com e sem gamificação. A rede social apresenta resultados melhorem nas tarefas, no exame final e na participação. A gamificação possui o pior desempenho no exame final e na participação, sendo melhor apenas nas tarefas em relação ao grupo de controle. Auvinen, Hakulinen e Malmi (2015) comparam ambientes com e sem gamificação, e heatmap, tendo como resultado maior ganho de pontos pelo grupo de gamificação, porém não significativo. Os estudantes do grupo que utilizou a gamificação obteve com mais frequência a pontuação máxima e entregou as tarefas antes do prazo final. Portanto, com a análise desses resultados a $\mathbf{Q P}_{3}$ é respondida.

\section{Conclusão}

O objetivo desse estudo foi identificar na literatura trabalhos que expliquem como é realizado o processo de avaliação de um ambiente com gamificação. Para atingir tal objetivo, foi desenvolvido um mapeamento sistemático. Desta forma, foram definidas três questões de pesquisa a serem respondidas. QP1: Como é realizada a comparação e a avaliação dos ambientes educacionais com e sem gamificação? QP2: Como é realizado o processo de avaliação dos ambientes? QP3: Quais são os pontos positivos e negativos da utilização de ambientes com gamificação?

De acordo com os resultados, há maioria dos trabalhos faz comparação de ambiente com e sem gamificação, porém também há comparação da heatmap e redes sociais (QP1). As atividades que compõe o processo de avaliação dos trabalhos, na maioria dos casos, são: exercícios/tarefas, pesquisa de satisfação e testes $\left(\mathbf{Q P}_{\mathbf{2}}\right)$, mas há também teste final, fórum de discussão, download de materiais e projeto. Além disso, verificou-se que a gamificação contribui positivamente, principalmente, na motivação, no acerto dos exercícios ou tarefas, na assiduidade, na satisfação e no engajamento. A nota (ou pontuação) foi o aspecto mais citado como resultado positivo e negativo $\left(\mathbf{Q P} \mathbf{P}_{3}\right)$. Apesar disso, muitos dos resultados obtidos com a aplicação da gamificação não são comprovados estatisticamente.

Portanto, não há um padrão para a execução da avaliação da gamificação, mas os exercícios ou tarefas são bastante utilizados e a partir deles podem ser observadas diferentes métricas, tais como acerto, conclusão, tempo de resposta, prazo de entrega e nota/pontuação. Também podem ser aplicados questionários para identificar a motivação, engajamento e satisfação dos estudantes.

A partir do estudo da arte da gamificação, serão analisados outros aspectos da gamificação não aprofundados neste trabalho (e.g., escolaridade, utilização de ambientes próprios) e a avaliação da gamificação em um ambiente educacional como trabalhos futuros.

\section{Referências}

Amriani, A., Indonesia, U., Aji, A. F., et al. (2013). An Empirical Study of Gamification Impact on E-Learning Environment. In: Computer Science and Network Technology (ICCSNT), 2013 3rd International Conference on. IEEE, 2013. p. 265-269. 
V Congresso Brasileiro de Informática na Educação (CBIE 2016)

Anais do XXVII Simpósio Brasileiro de Informática na Educação (SBIE 2016)

Attali, Y. and Arieli-Attali, M. (2015). Gamification in assessment: Do points affect test performance? Computers and Education, v. 83, p. 57-63.

Auvinen, T., Hakulinen, L. and Malmi, L. (2015). Increasing Students' Awareness of Their Behavior in Online Learning Environments with Visualizations and Achievement Badges. IEEE Transactions on Learning Technologies, v. 8, n. 3, p. 261-273.

Bissolotti, K., Nogueira, H. G. and Pereira, A. T. C. (2014). Potencialidades das mídias sociais e da gamificação na educação a distância. RENOTE, v.12, n.2.

Burke, B. Gamify: How gamification motivates people to do extraordinary things. Bibliomotion, 2014.

De-Marcos, L., Domínguez, A., Saenz-De-Navarrete, J. and Pagés, C. (2014). An empirical study comparing gamification and social networking on e-learning. Computers and Education, v. 75, p. 82-91.

Denny, P. (2013). The effect of virtual achievements on student engagement. Proceedings of the SIGCHI Conference on Human Factors in Computing Systems - CHI '13, p. 763-772.

Deterding, S., Dixon, D., Nacke, L.E., Khaled, R. (2011). Gamification: Toward a definition. In: Conference on Human Factors in Computing Systems, pp. 9-15, ACM.

Fardo, M. L. (2013). A Gamificação aplicada em Ambientes de Aprendizagem. RENOTE, v. 11, n. 1, p. 1-9.

Hew, K. F., Huang, B., Chu, K. W. S. and Chiu, D. K. W. (2016). Engaging Asian students through game mechanics: Findings from two experiment studies. Computers and Education, v. 92-93, p. 221-236.

Johnson, L.; Adams, S.; Cummins, M.; Estrada, V.; Freeman, A.; Ludgate, H. (2013). The NMC Horizon Report: 2013 Higher Education Edition. New Media Consortium.

Klock, A. C. T., Carvalho, M. F. De, Rosa, B. E. Da and Gasparini, I. (2014). Análise das técnicas de Gamificação em Ambientes Virtuais de Aprendizagem. RENOTE - Revista Novas Tecnologias na Educação, v. 12, n. 2, p. 1-10.

Klock, A. C. T., Gasparini, I., Kemczinski, A., Hounsell, M. and Isotani, S. (2015). One man's trash is another man's treasure: um mapeamento sistemático sobre as características individuais na gamificação de ambientes virtuais de aprendizagem. In: Anais do Simpósio Brasileiro de Informática na Educação, v. 26, n. 1, p. 539-548.

Krause, M., Mogalle, M., Pohl, H. and Williams, J. J. (2015). A Playful Game Changer: Fostering Student Retention in Online Education with Social Gamification. Proceedings of the 2nd ACM Conference on Learning@ Scale (L@S'15), p. 95-102.

O'Donovan, S., Gain, J., Marais, P. (2013). A case study in the gamification of a universitylevel games development course. In Proceedings of the South African Institute for Computer Scientists and Information Technologists Conference, p. 242-251, ACM.

Pedro, L. Z., Lopes, A. M. Z., Prates, B. G., Vassileva, J. and Isotani, S. (2015). Does gamification work for boys and girls? Proceedings of the 30th Annual ACM Symposium on Applied Computing - SAC '15, p. 214-219.

Petersen, K., Vakkalanka, S., Kuzniarz, L. (2015). Guidelines for conducting systematic mapping studies in software engineering: An update. In: Information and Software Technology, v. 64, pp. 1-18, Elsevier.

Sousa Borges, S. de, Durelli, V. H., Reis, H. M., Isotani, S. (2014). A systematic mapping on gamification applied to education. In Proceedings of the 29th Annual ACM Symposium on Applied Computing, p. 216-222, ACM

$\mathrm{Su}, \mathrm{C}$. H. and Cheng, C. H. (2015). A mobile gamification learning system for improving the learning motivation and achievements. Journal of Computer Assisted Learning, v. 31, n. 3, p. $268-286$.

Werbach, K.; Hunter, D. For the win: How game thinking can revolutionize your business. Wharton Digital Press, 2012. 